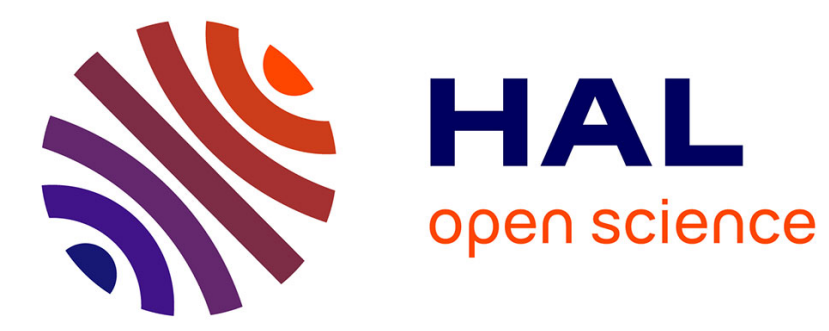

\title{
L'enseignement des langues kanak en Nouvelle-Calédonie Jacques Vernaudon
}

\section{To cite this version:}

Jacques Vernaudon. L'enseignement des langues kanak en Nouvelle-Calédonie. Hermès, La Revue Cognition, communication, politique, 2013, 65, p. 119-125. hal-01777639

\section{HAL Id: hal-01777639 \\ https://hal.science/hal-01777639}

Submitted on 23 Feb 2019

HAL is a multi-disciplinary open access archive for the deposit and dissemination of scientific research documents, whether they are published or not. The documents may come from teaching and research institutions in France or abroad, or from public or private research centers.
L'archive ouverte pluridisciplinaire HAL, est destinée au dépôt et à la diffusion de documents scientifiques de niveau recherche, publiés ou non, émanant des établissements d'enseignement et de recherche français ou étrangers, des laboratoires publics ou privés. 


\title{
L'ENSEIGNEMENT DES LANGUES KANAK EN NOUVELLE- CALÉDONIE
}

\author{
Jacques Vernaudon
}

\author{
C.N.R.S. Editions | «Hermès, La Revue »
}

2013/1 n 65 | pages 112 à 118

ISSN 0767-9513

ISBN 9782271076809

Article disponible en ligne à l'adresse :

https://www.cairn.info/revue-hermes-la-revue-2013-1-page-112.htm

Distribution électronique Cairn.info pour C.N.R.S. Editions.

(C) C.N.R.S. Editions. Tous droits réservés pour tous pays.

La reproduction ou représentation de cet article, notamment par photocopie, n'est autorisée que dans les limites des conditions générales d'utilisation du site ou, le cas échéant, des conditions générales de la licence souscrite par votre établissement. Toute autre reproduction ou représentation, en tout ou partie, sous quelque forme et de quelque manière que ce soit, est interdite sauf accord préalable et écrit de l'éditeur, en dehors des cas prévus par la législation en vigueur en France. Il est précisé que son stockage dans une base de données est également interdit. 


\section{L'enseignement des langues kanak en Nouvelle-Calédonie}

\section{Les langues kanak dans le paysage linguistique calédonien}

Au moment du premier contact avec l'Occident en 1774, l'archipel calédonien comptait probablement plus d'une trentaine de langues autochtones, sans qu'aucune d'entre elles ne soit véhiculaire. De tradition orale, elles ont pour la plupart été transmises jusqu'à nos jours et portent désormais le nom de «langues kanak». Elles appartiennent au sous-groupe océanien de la famille des langues austronésiennes (Moyse-Faurie, Rivierre et Vernaudon, 2012). En 2009, 66884 personnes de 14 ans et plus déclaraient parler une langue kanak, soit $36 \%$ des habitants de cette tranche d'âge (ISEE, 2009).

Le morcellement linguistique de la NouvelleCalédonie est caractéristique de la région. Il est lié d'une part à l'ancienneté du peuplement, les langues contemporaines étant issues, à l'exception du fagauvea, de la diversification dialectale à partir d'une protolangue océanienne commune parlée par les premiers navigateurs austronésiens arrivés dans l'archipel il y a environ 3000 ans; d'autre part, il fut structurellement induit par le fonctionnement de l'ancienne société kanak. En l'absence de pouvoir politique centralisé, aucun groupe ethnolinguistique n'avait instrumentalisé sa propre langue pour asseoir sa domination sur les autres. La multiplicité des langues participait d'un équilibre sociolinguistique utile à l'échange entre groupes exogames et virilocaux, le principe étant, dans l'idéal, le suivant: un homme du groupe A va chercher son épouse dans un groupe B qui parle une langue distincte de la sienne. Les époux reviennent résider dans le groupe A du mari, mais l'épouse transmet sa langue B à ses enfants, en prévision d'échanges coutumiers à venir avec le clan utérin. Les enfants deviennent donc bilingues. À défaut de langue véhiculaire, le plurilinguisme des individus, parlant a minima la langue de leur clan paternel et celle de leur clan utérin, permettait la communication entre les différents ensembles ethnolinguistiques. Maurice Leenhardt (1946) rappelle à ce sujet que «la possession de plusieurs langues [était] l'un des éléments essentiels de la culture personnelle de l'ancien Canaque».

À cette diversité endogène s'ajoutent, dans le paysage linguistique calédonien contemporain, toutes les langues 
issues de migrations plus récentes, à commencer par le français, désormais seule langue officielle et principale langue de scolarisation. C'est aussi la seule langue véhiculaire. $97 \%$ de la population calédonienne de 14 ans et plus déclare le parler, le lire et l'écrire (ISEE, 2009).

\section{L'entreprise missionnaire : premières alphabétisations}

L'entrée des langues kanak à l'école débute avec les premières alphabétisations en langues vernaculaires entreprises par les missionnaires protestants. Cette œuvre participera à la pérennisation des quelques langues choisies pour l'évangélisation et marquera durablement les esprits dans le monde kanak en apportant la contradiction au déni de fonction didactique imposé par la suite par l'école coloniale. Parmi les langues les plus enseignées, on trouve encore aujourd'hui le drehu, le nengone et l'ajië, principales langues d'évangélisation protestante.

La Société missionnaire de Londres (London Missionary Society, LMS), fondée en 1795, choisit Tahiti comme première implantation dans le Pacifique en 1797. La stratégie d'évangélisation protestante est alors inspirée des principes formulés par le luthérien Bartholomaüs Ziegenbalg au début du XVIII ${ }^{\mathrm{e}}$ siècle (Nicole, 1988):

Église et école vont de pair. Tout chrétien devrait être à même de lire la Parole de Dieu: donc tous les enfants chrétiens doivent recevoir une instruction. Cette parole doit leur être présentée dans leur propre langue.

De fait, un premier catéchisme fut publié en tahitien dès 1801 et la première édition complète de la Bible en tahitien parut en 1838. Outre le travail de normalisation orthographique et de traduction, les missionnaires vont déployer une intense activité d'alphabétisation vernacu- laire. En 1823, le commandant Duperrey écrit dans son rapport au ministre de la Marine et des Colonies à propos des écoles missionnaires: "Tous les naturels de Tahiti savent lire et écrire» (Nicole, 1988).

L'œuvre missionnaire protestante se poursuit vers l'est du Pacifique et arrive en Nouvelle-Calédonie. Après une première tentative infructueuse dans le sud de la Grande Terre et à l'île des Pins, la LMS va s'implanter durablement aux îles Loyauté à partir des années 1840. Un rapport du délégué de Salins, en 1885, adressé au gouverneur Le Bouchet signale à propos de l'île de Maré que «toute la population qui n'a pas dépassé 27 ans sait lire et écrire le maréen. Il faut être de mauvaise foi pour nier les résultats obtenus par les pasteurs anglais» (Leenhardt, 1980).

La France prend possession de la Nouvelle-Calédonie en 1853. Lors d'une expédition à Lifou en juin 1864 destinée à neutraliser l'influence des missionnaires anglosaxons et à imposer l'autorité de la France sur l'ensemble de l'île, le gouverneur Guillain fait "arrêter et conduire à bord du Fulton, à titre d'otages, toutes les femmes samoa et indigènes réfugiées chez le pasteur Mac Farlane, au nombre desquelles se trouvait la propre femme du grand chef Boula » (Dauphiné, 1990). Le gouverneur veut se montrer conciliant et autorise l'épouse du grand chef à écrire à son mari. Joël Dauphiné (1990) nous livre la description de la scène par Guillain lui-même ${ }^{1}$ :

C'était la première fois que je voyais une femme à type si voisin de la race nègre, posée avec tant d'aisance devant une table à écrire, la physionomie pensive et réfléchie, inscrivant elle-même sur le papier, ses idées, ses réflexions et traçant une ligne de conduite à son mari, grand chef de Loessi. Je ne pouvais m'empêcher de me dire tristement, qu'aujourd'hui encore, après dix ans de notre occupation, il serait impossible de trouver sa pareille dans toute la Nouvelle-Calédonie ${ }^{2}$; et à ce sujet il faut bien reconnaître que si les missionnaires protestants 
ont fait beaucoup de mal dans le but de détacher de nous les indigènes des Loyalty, ils auront notablement contribué à la première éducation d'un grand nombre de ceux-ci.

\section{L'école coloniale et l'impérialisme linguistique}

Le même Guillain s'employa pourtant avec force à interdire l'usage des langues kanak dans les écoles missionnaires pour imposer le français. Un arrêté du 15 octobre 1863 dispose que dans les écoles privées «l'enseignement portera sur: L'instruction morale et religieuse, la lecture, l'écriture, les éléments de la langue française, le calcul et le système légal des poids et mesures» et que "l'étude des idiomes calédoniens est formellement interdite dans toutes les écoles». Ces dispositions avaient pour objectif, selon Guillain, de «faciliter nos relations avec les indigènes et l'accomplissement de notre mission civilisatrice à leur égard, ce qui exige, avant tout, qu'ils nous comprennent». L'arrêté du 3 août 1905 précisant le fonctionnement des écoles primaires réaffirma à son article 12 que «le français sera seul en usage à l'école». Les langues kanak furent également interdites de publication en 1921 (Rivierre, 1985).

Cette étape marque un bouleversement majeur des équilibres sociolinguistiques, puisqu'elle conduira d'un bilinguisme égalitaire dans la société kanak ancienne (Haudricourt, 1961) à un impérialisme monolingue dans la société coloniale en formation. Ce paradigme idéologique ne sera jamais démenti jusqu'en 1984. Marie Salaün (2005a) souligne cependant le caractère velléitaire de la politique coloniale de francisation ${ }^{3}$ : jamais l'assimilation, qui implique la maîtrise de la langue de la Mère Patrie, n'a été sérieusement envisagée pour des Kanak appelés, selon les termes de l'époque, «à s'effacer devant les peuples supérieurs".
C'est paradoxalement après la Seconde Guerre mondiale, avec la fin du régime de l'indigénat, que le processus d'assimilation devient efficace dans l'arasement des particularismes locaux. L'école, avec la généralisation de la scolarisation primaire puis secondaire, l'homogénéisation des enseignements, la disparition d'une scolarisation indigène spécifique et l'alignement sur ce qui se fait en Métropole, va puissamment contribuer à l'imposition du «tout-français » jusqu'au début des «événements » ${ }^{4}$ en 1984.

\section{La décolonisation et les langues kanak}

Outre les questions foncières, l'éducation est un thème majeur dans les revendications indépendantistes qui émergent à partir de la fin des années 1960. Salaün et Vernaudon (2009) rappellent le contexte dans lequel sont formulées les premières récriminations autour de l'école «coloniale»:

$\mathrm{Au}$ lendemain de la Seconde Guerre mondiale, les conséquences de l'accession des Kanak à la citoyenneté française auraient dû être, en toute hypothèse, très importantes au plan scolaire. L'extension de l'obligation scolaire jusqu'alors applicable aux seuls colons, l'abolition de la distinction entre écoles européennes et écoles indigènes, et l'égalité de moyens théorique (qui passe aussi par une uniformisation des contenus enseignés) généralisent l'accès des enfants kanak aux écoles primaires (années 1950) puis au collège d'enseignement secondaire (années 1970). Pour autant, cette "massification» ne s'accompagne pas d'une démocratisation: alors qu'il leur est théoriquement possible de se présenter aux examens longtemps réservés aux seuls Blancs, la part des diplômés dans la population kanak reste infime. Un vaste processus d'élimination fait d'abandons et de relégation vers les voies professionnelles les 
moins valorisées fait qu'à la fin des années 1970, si les enfants kanak représentent $55 \%$ des effectifs du primaire, ils ne représentent plus que $20 \%$ des effectifs de la classe de fin de secondaire, et $10 \%$ des reçus au titre qui permet l'accès aux études supérieures, le baccalauréat (Kohler et Wacquant, 1985). Cent vingt ans après la prise de possession par la France de la Nouvelle-Calédonie, neuf Mélanésiens sur dix n’ont donc aucun diplôme.

La première demande officielle d'une prise en compte de la spécificité mélanésienne dans l'enseignement date de 1971, lorsque des élus locaux réclament l'application au territoire de la loi Deixonne, qui régit en Métropole depuis 1951 l'enseignement des langues régionales de France. Consulté sur la recevabilité de la demande, le vice-recteur en poste à Nouméa répond en 1975 que la pratique de la langue française doit être « constante » à l'école primaire et que «la présence de langues vernaculaires mélanésiennes parmi les épreuves de baccalauréat ne saurait, à brève ou moyenne échéance, être valablement envisagée» (Salaün et Vernaudon, 2011).

Face à ce refus, la question de la place des langues kanak et du français à l'école, va être rapidement investie d'une dimension politique. Dans le camp indépendantiste, en 1984, une des premières décisions de la majorité territoriale nouvellement élue et dirigée par Jean-Marie Tjibaou, est d'abroger l'ensemble des dispositions héritées de la colonisation interdisant l'usage des langues kanak à l'école et dans les publications ${ }^{5}$. Un an plus tard, les indépendantistes lancent un mot d'ordre de boycott des écoles «coloniales» qui voit la création, éphémère, d'écoles communautaires, les écoles populaires kanak (EPK) où l'enseignement se fait en langues vernaculaires (Gauthier, 1996). Dans le camp loyaliste, la revendication de reconnaissance des langues kanak est assimilée à une contestation de la présence de la France et de ses ressortissants, voire à une menace pour l'intégrité de la République (Salaün et Vernaudon, 2011).
À l'issue des «événements", le consensus autour de la nécessité d'un retour à la paix civile, exprimé par la signature des accords de Matignon-Oudinot en 1988, implique une réponse des institutions aux revendications culturelles des nationalistes kanak. Les provinces ${ }^{6}$ obtiennent la possibilité de procéder à "l'adaptation des programmes en fonction des réalités culturelles et linguistiques» dans le premier degré avec un quota de cinq heures hebdomadaires. La loi Deixonne est étendue à la Nouvelle-Calédonie et quatre langues kanak sont introduites dans les épreuves du baccalauréat en 1992. L'accord de Nouméa (1998) offre des perspectives de réforme éducative plus ambitieuses en prévoyant le transfert immédiat des compétences pour les programmes de l'enseignement primaire, la formation des maîtres et le contrôle pédagogique. Il dispose que «les langues kanak sont, avec le français, des langues d'enseignement et de culture». Une filière universitaire de Langues et cultures régionales (LCR) est créée à l'université de Nouvelle-Calédonie en 1999.

\section{Expérimentations locales et recherche internationale}

Cette création a encouragé l'essor local d'une recherche universitaire sur le bilinguisme précoce à l'école, étayée par les données scientifiques internationales ${ }^{7}$.

Un premier dispositif expérimental d'enseignement bilingue est mis en place de 2003 à 2005 à la demande du gouvernement de la Nouvelle-Calédonie, dans dix écoles, auprès d'un effectif total de 210 élèves, en petite, moyenne et grande sections de maternelle et en cours préparatoire. Les évaluations, conduites par des psycholinguistes de l'université de Nantes, ont confirmé que les élèves qui participaient à 5 heures hebdomadaires d'enseignement en langue kanak, pris sur le volume globale des 27 heures hebdomadaires d'enseignement (ce qui équivalait donc 
à 5 heures «de moins» en français), avaient progressé "massivement» en langue kanak tout en conservant un niveau équivalent en français à celui des élèves qui étaient scolarisés exclusivement en français (Nocus, Florin et Guimard, 2005). Des évaluations complémentaires ont attesté de phénomènes de transferts positifs vers le français à partir du cours préparatoire (Nocus, Florin, Guimard et Vernaudon, 2007). Cet enseignement a reçu en outre un accueil enthousiaste de la part des parents kanak qui y voyaient un moyen d'éviter une rupture avec les « racines » et de favoriser l'épanouissement de leurs enfants grâce à une valorisation de leur vécu en établissant un rapport harmonieux entre les langues (Salaün, 2005b).

Une autre étude, menée en 2010 à la demande du gouvernement de la Nouvelle-Calédonie (Gauchon, 2010), conclut que «les données étudiées traduisent un effet plutôt positif de l'enseignement LCK sur la réussite scolaire telle qu'elle est mesurée à partir des évaluations $\mathrm{CP}$ ».

Étayés par ces résultats positifs, les programmes scolaires calédoniens prévoient depuis 2005, pour les élèves dont les parents en ont exprimé le vœu, un enseignement des langues kanak à raison de 7 heures hebdomadaires en maternelle et de 5 heures aux cycles 2 et 3 . Les finalités annoncées sont de trois ordres. Il s'agit de participer au côté des familles à la transmission du patrimoine linguistique et culturel local; cet enseignement doit permettre l'amélioration des résultats scolaires des élèves dont ce sont les langues maternelles ou d'origine en favorisant leur épanouissement affectif et intellectuel et le renforcement de leurs compétences langagières; enfin, il est attendu que cet enseignement favorise le «vivre ensemble». En 2012, environ 5700 élèves de l'école primaire publique et sous contrat participaient à des enseignements LCK (soit $16 \%$ des 35000 élèves du premier degré). Dix-neuf langues kanak étaient enseignées. Les données sont incomplètes pour le second degré, mais l'enseignement LCK, dispensé dans dix langues kanak, y concernait au moins 1700 élèves sur une population scolaire totale de 32500 (soit $5 \%)^{8}$.

\section{Un avenir incertain, un dossier en tension}

Ces avancées dans l'espace scolaire ne doivent cependant pas occulter la fragilisation de la transmission intergénérationnelle. Selon l'atlas interactif des langues du monde en danger, 13 des 28 langues kanak sont menacées (Moseley, 2010). Une enquête (Veyret et Gobber, 2000) intitulée «Quelles langues parlent nos élèves à l'entrée en $6^{\mathrm{e}}$ ?», soulignait déjà en 2000 la prégnance du français:

Une forte proportion d'élèves (70 \%) affirme avoir parlé le français en premier. Ce résultat est particulièrement révélateur d'une forte évolution récente en faveur du français, lorsqu'on le met en relation avec la langue maternelle des parents : $33,3 \%$ des pères et $34,7 \%$ des mères seulement sont de langue maternelle française. Il y a donc une nette progression, dans les relations parents-enfants de la génération des "Accords", du français comme langue de communication et une volonté de l'utiliser, sans doute en vue de favoriser la réussite scolaire des enfants.

Plus récemment Véronique Fillol et Claire Colombel (à paraître) confirment l'érosion linguistique, en particulier en milieu urbain, et soulignent le poids de l'idéologie monolingue 9 de l'école «qui vient perturber la transmission des langues familiales». Par ailleurs, près des deux tiers de la population totale calédonienne se concentre à Nouméa et dans sa périphérie. Or la ville favorise la mixité ethnique et sociale, mais aussi le choix exclusif de la langue dominante, le français, comme langue de communication quotidienne, y compris dans le giron familial.

À ces facteurs systémiques s'ajoute une contrainte conjoncturelle. À l'approche des échéances politiques de $2014-2018^{10}$, on constate un nouveau raidissement autour de la question des langues kanak à l'école où ressurgissent les 
anciens clivages. Les langues sont des instruments de communication, mais aussi des étendards identitaires. Les partis loyalistes, attentifs à la crispation de leur base électorale à l'approche du référendum d'autodétermination, rechignent à soutenir le dossier de l'enseignement des langues kanak et à généraliser les dispositifs existants de peur d'être soupçonnés de compromission avec l'adversaire indépendantiste, porteur historique de la revendication culturelle.

\section{NOTES}

1. Extrait du rapport du 5 juillet 1864. Le rapport précise que la lettre adressée au grand chef par son épouse, écrite exclusivement en drehu, faisait quatre pages.

2. La «Nouvelle-Calédonie» désigne ici uniquement la Grande Terre, par opposition aux «Loyalty», les îles Loyauté.

3. Selon Salaün (2005a), la part du budget de la colonie consacré aux écoles indigènes - supposées pourtant être le principal vecteur de la francisation - oscille entre 0,3 et $0,7 \%$.

4. C'est ainsi que l'on nomme pudiquement les quatre années de quasi guerre civile qui ont embrasé la Nouvelle-Calédonie à partir de 1984

5. Délibération $\mathrm{n}^{\circ} 333$ de la commission permanente de l'Assemblée territoriale du 29 février 1984.

6. La Nouvelle-Calédonie a été divisée en 1989 en trois provinces: sud, nord et îles Loyauté, les deux dernières étant de majorité indépendantiste depuis cette date.

\section{RÉFÉRENCES BIBLIOGR A PHIQUES}

Cummins, J., "L'éducation bilingue: qu'avons-nous appris de cinquante ans de recherche?", in Nocus, I., Vernaudon, J. et PAIA, M. (dir.), Apprendre plusieurs langues, plusieurs langues pour apprendre: l'école plurilingue en Outre-mer, Rennes, Presses universitaires de Rennes, à paraître.

DAUPHINÉ, J., Lifou (1864): la prise de possession, Nouméa, CTRDP, 1990.
Même si de nombreux acteurs de terrain poursuivent leurs efforts pour la promotion des langues kanak dans les écoles, les résistances politiques et le morcellement institutionnel de la Nouvelle-Calédonie entravent les synergies à l'échelle du pays, alors même que ce dernier est doté de compétences élargies et qu'il dispose de ressources institutionnelles inégalées comparativement aux autres collectivités françaises pour promouvoir les langues et les cultures locales.

7. Pour une revue sur ce point, voir Cummins (à paraître).

8. Source: Fabienne Ixeko, service de l'enseignement des langues et de la culture kanak, vice-rectorat de la Nouvelle-Calédonie.

9. Nous désignons par ce terme un ensemble de représentations négatives sur le bilinguisme précoce des élèves océaniens qui agrègent, d'une part, les notions de surcharge cognitive, de mélange des langues et de confusion mentale, et d'autre part, des conceptions dépréciatives sur les langues océaniennes (ces langues seraient "pauvres", "compliquées à prononcer», "trop nombreuses», "sans grammaire», «dénuées d'abstraction», "peu précises», etc.). Pour une critique de ces représentations, $c f$. Vernaudon (2005) et Cummins (à paraître).

10. Entre 2014 et 2018 , les électeurs calédoniens devront décider du maintien ou non de la Nouvelle-Calédonie dans la France.

Fillol, V. et ColOMbel, C., «Quelle(s) politique(s) linguistique(s) éducative(s) pour la Nouvelle-Calédonie? Quels dispositifs d'enseignement des langues? Réflexions sociodidactiques", in Nocus, I. Vernaudon, J. et PAiA, M. (dir.), Apprendre plusieurs langues, plusieurs langues pour apprendre: l'école plurilingue en Outre-mer, Rennes, Presses universitaires de Rennes, à paraître. 
GAUCHON, D., Étude statistique des résultats aux évaluations $C P$ des élèves ayant suivi un enseignement LCK en cycle 1, rapport non publié, 2010.

GAUTHIER, J., Les Écoles populaires kanak. Une révolution pédagogique?, Paris, L'Harmattan, 1996.

HAUDRICOURT, A.-G., «Richesse en phonèmes et richesse en locuteurs", L'Homme, nº 1, 1961, p. 5-10.

ISEE, Recensement général de la population, Nouméa, ISEE, 2009.

Kohler, J.-M. et WACQUANT, L. J.-D., L'École inégale. Éléments pour une sociologie de l'école en Nouvelle-Calédonie, Nouméa, Institut culturel mélanésien, ORSTOM, 1985.

LeenhardT, M., Langues et dialectes de l'Austro-Mélanésie, Paris, Institut d'ethnologie, 1946

LeEnhardt, R., Au vent de la Grande Terre, Les Îles Loyalty de 1840 à 1895, Paris, Imprimerie Claude Bernard, 1980.

Lindholm-Leary, K. J. et Borsato, G., «Academic Achievement », in Genesee, F., Lindholm-LeARY, K., SAUNDERS, W. et Christian, D. (dir.), Educating English Language Learners, New York, Cambridge University Press, 2006, p. 176-222.

Moseley, C. (dir.), Atlas of the World's Languages in Danger, Paris, Unesco, 2010 [3ééd.].

Moyse-Faurie, C., Rivierre, J.-C. et Vernaudon, J., «Les langues kanak», in Bonvalot, J. et GAY, J.-C. (dir.), Atlas de la Nouvelle-Calédonie, Nouméa, IRD, 2012, p. 119-122.

Nicole, J., Au pied de l'écriture, Histoire de la traduction de la Bible en tahitien, Papeete, Haere pō no Tahiti, 1988.

Nocus, I., Florin, A. et Guimard, P., Rapport d'évaluation de l'expérimentation "L'enseignement des langues et de la culture kanak à l'école primaire de la Nouvelle-Calédonie», Axe psycholinguistique, Labécd, Université de Nantes, non publié, 2005.
Nocus, I., Florin, A., Guimard, P. et Vernaudon, J., «Effets d'un enseignement en langue kanak sur les compétences oral/écrit en français au cycle 2 en Nouvelle-Calédonie», Bulletin de psychologie, $\mathrm{n}^{\circ} 491$, vol. 5, 2007, p. 471-488.

RivierRe, J.-C., «La colonisation et les langues en Nouvelle-Calédonie», Les temps modernes, dossier "Nouvelle-Calédonie: Pour l'indépendance», n 464, mars 1985, p. 1688-1717.

SAlaÜN, M., L'École indigène, Nouvelle-Calédonie, 1885-1945, Rennes, Presses universitaires de Rennes, 2005a.

SAlaüN, M., Rapport d'évaluation de l'expérimentation "L'enseignement des langues et de la culture kanak à l'école primaire publique de la Nouvelle-Calédonie», Axe sociolinguistique, département des Sciences de l'éducation, Université Paris 5, non publié, $2005 b$.

SALAÜN, M. et Vernaudon, J., «"Les langues kanak sont, avec le français, des langues d'enseignement et de culture en NouvelleCalédonie.", Et après?», in Clairis, C., Costaouec, D., Coyos, J.-B. et JEANNOT-FOURCAUD, B. (dir.), Langues et cultures régionales de France, Dix ans après, Cadre légal, politiques, médias, Paris, L'Harmattan, 2011, p. 129-151.

SAlaÜN, M. et VernaUdon, J., «La citoyenneté comme horizon: destin commun, demande sociale et décolonisation de l'École en Nouvelle-Calédonie aujourd'hui», Anthropologie et sociétés, vol. 33 , n 2, Québec, Université de Laval, 2009, p. 63-80.

VERNAUDON, J., «Des représentations sur les langues océaniennes aux options pour leur enseignement", in FILLOL, V. et VERNAUDON, J. (dir.), Stéréotypes et représentations en Océanie, Actes du $17^{e}$ colloque Corail, Nouméa, Corail, 2005, p. 77-99.

Veyret, M. P. et Gobber M.-J., Quelles langues utilisent nos élèves?: analyse d'une enquête faite auprès des élèves de sixième des collèges publics de Nouvelle-Calédonie, Nouméa, CNDP, IUFM du Pacifique et vice-rectorat de Nouvelle-Calédonie, 2000. 